\title{
Propriedades Mecânicas de Nanocompósitos de Polipropileno e Montmorilonita Organofílica
}

\author{
Lucilene B. de Paiva, Ana R. Morales \\ Faculdade de Engenharia Química, UNICAMP \\ Thiago R. Guimarães
Departamento de Engenharia Metalúrgica e de Materiais, Escola Politécnica, USP-SP
}

Resumo: Neste trabalho foram preparados nanocompósitos de polipropileno e montmorilonita organofílica comercial Cloisite $20 \mathrm{~A}$ em concentrações de $2,5 \%, 5,0 \%, 7,5 \%$ e 10,0\% e polipropileno graftizado com anidrido maleico como agente compatibilizante pela técnica de intercalação do fundido em extrusora de dupla rosca. Os materiais obtidos foram caracterizados por difração de raios x, ensaios de tração e impacto de acordo com as normas ASTM D638 e D256, respectivamente. Os resultados de difração de raios x indicaram a formação de nanocompósitos com estruturas parcialmente esfoliadas ou intercaladas, dependendo da composição, que proporcionaram aumento do módulo de elasticidade enquanto as propriedades de resistência ao impacto não foram satisfatórias.

Palavras-chave: Nanocompósitos, montmorilonita, polipropileno, polipropileno graftizado com anidrido maleico.

\section{Mechanical Properties of Polypropylene and Organophilic Montmorillonite Nanocomposites}

Abstract: Nanocomposites of polypropylene and commercial montmorillonite organophilic Cloisite 20A containing 2.5\%, $5 \%, 7.5 \%$ and $10 \%$ of clay with polypropylene-graft-maleic anhydride as coupling agent were prepared through the melt intercalation by twin-screw extruder. The materials were characterized by $\mathrm{x}$-ray diffraction, tensile and impact properties according to ASTM D638 and D256 test methods, respectively. The x-ray diffraction results showed exfoliated or intercalated structures for different concentrations of the clay. The tensile modulus improved with the increase of the amount of the clay while the impact strength showed unsatisfactory results.

Keywords: Nanocomposites, montmorillonite, polypropylene, polypropylene-graft-maleic anhydride.

\section{Introdução}

Nanocompósitos constituem uma classe de materiais formados por híbridos de materiais orgânicos e inorgânicos, onde a fase inorgânica está dispersa em nível nanométrico em uma matriz polimérica. Esta classe de materiais começou a ser estudada na década de 80 pelo Laboratório de Pesquisa da Toyota com o desenvolvimento de nanocompósitos de poliamida e argila ${ }^{[1-3]}$.

A fase inorgânica mais utilizada na preparação de nanocompósitos poliméricos é a argila montmorilonita de origem natural, cuja razão de aspecto é bastante elevada. Apresenta boa capacidade de delaminação somada à alta resistência a solventes e estabilidade térmica necessária aos processos de polimerização e de extrusão, fatores que levaram à sua popularização como carga para nanocompósitos com eficácia comprovada.

Essa argila pertence ao grupo estrutural dos filossilicatos 2:1 e, conforme mostrado na Figura 1, é composta por camadas estruturais constituídas por duas folhas tetraédricas de sílica, com uma folha central octaédrica de alumina, que se mantêm unidas por átomos de oxigênio comuns a ambas folhas que apresentam espessura que pode chegar a 1 nanômetro e dimensões laterais que podem variar de $300 \AA$ a vários microns. A montmorilonita em seu estado natural tem caráter hidrofílico e para haver uma boa dispersão em matrizes poliméricas é necessário torná-la organofílica através de um processo de modificação superficial, onde são trocados os cátions sódio presentes em lacunas (também chamadas de galerias) existentes entre as camadas estruturais, por cátions de sais quaternários de amônio de cadeias longas contendo acima de 12 carbonos, que proporcionam a expansão entre as galerias facilitando assim a incorporação das cadeias poliméricas ${ }^{[4-6]}$.

Três principais tipos de estruturas que estão apresentadas na Figura 2 podem ser obtidas quando uma argila é dispersa em uma matriz polimérica: estrutura de fase separada quando as cadeias poliméricas não intercalam as camadas de argila levando à obtenção de uma estrutura de propriedades similares às de um compósito convencional; estrutura intercalada quando as cadeias poliméricas são intercaladas entre as camadas de argila, formando uma

Autor para correspondência: Ana R. Morales, Departamento de Tecnologia de Polímeros, FEQ, UNICAMP, Caixa Postal 6066, CEP 13083-970, Campinas, SP.E-mail:morales@dtp.feq.unicamp.br 


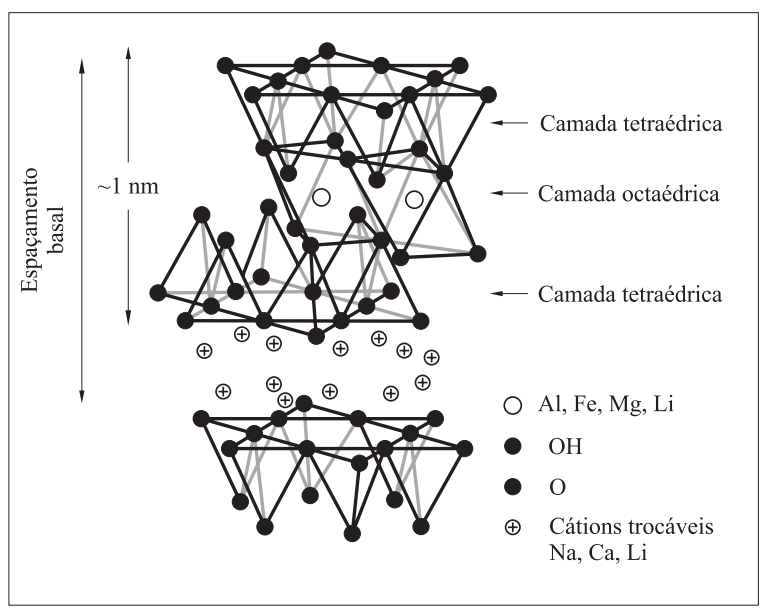

Figura 1. Estrutura dos filossilicatos $2: 1$ mostrando duas camadas de silicato de alumínio com cátions sódio nas galerias

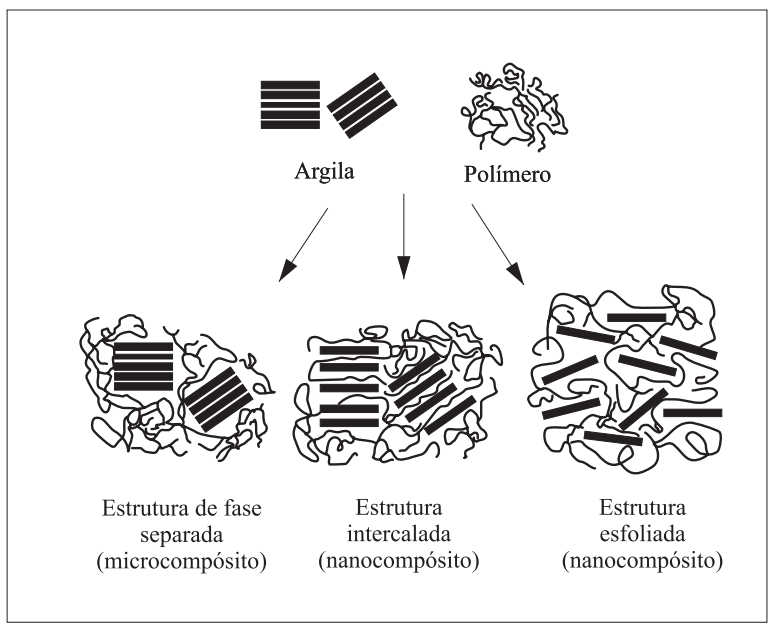

Figura 2. Estruturas dos nanocompósitos

estrutura multicamada bem ordenada, que apresenta propriedades superiores à de um compósito convencional; e estrutura esfoliada, onde a argila é completa e uniformemente dispersa em uma matriz polimérica, maximiza as interações polímero-argila e leva a significativas melhorias nas propriedades físicas e mecânicas ${ }^{[3,7,8]}$.

A uniforme dispersão da nanocarga na matriz polimérica produz uma larga interação interfacial, o que representa a característica peculiar do nanocompósito e o diferencia de um compósito convencional. Os nanocompósitos apresentam melhores propriedades mecânicas, térmicas, de barreira e retardância à chama quando comparados à matriz pura e aos compósitos convencionais.

Atualmente, diversas matrizes poliméricas como poli(metacrilato de metila), poliamida, polietileno, polipropileno, poliestireno, poli(tereftalato de etileno), poli(cloreto de vinila), copoli(acrilonitrila/butadieno/estireno), entre outras são empregadas na preparação de nanocompósitos com argilas para aplicações nas áreas automobilística, de embalagens, médica, de filmes anti-corrosão, de materiais têxteis, de liberação controlada de drogas por polímeros, et ${ }^{[9-12]}$.

Este trabalho descreve a preparação de nanocompósitos de polipropileno/polipropileno graftizado com anidrido maleico e montmorilonita organofílica por extrusão de dupla rosca e a caracterização estrutural por difração de raios-x e quanto às propriedades mecânicas de tração e impacto de acordo com as normas ASTM D638 ${ }^{[13]}$ e D256 ${ }^{[14]}$.

\section{Experimental}

\section{Materiais}

Os materiais utilizados na preparação dos nanocompósitos foram o polipropileno (PP) Prolen XM6150K da Polibrasil, polipropileno graftizado com anidrido maleico (PP-g-AM) OREVAC CA-100 da Atofina e a argila montmorilonita Cloisite 20A fornecida pela Southern Clay Products.

\section{Métodos}

Os nanocompósitos foram processados em uma extrusora dupla rosca co-rotante de $25 \mathrm{~mm}$, da marca Werner Pfeiderer, Modelo ZSK 25 Mega Compounder, L/D36, a $300 \mathrm{rpm} \mathrm{e}$ com perfil de temperaturas nas respectivas zonas de aquecimento de: $230{ }^{\circ} \mathrm{C}, 140{ }^{\circ} \mathrm{C}, 140^{\circ} \mathrm{C}, 150{ }^{\circ} \mathrm{C}$ e $160{ }^{\circ} \mathrm{C}$. Primeiramente foi preparado um masterbatch contendo $75 \%$ de polipropileno graftizado com anidrido maleico e $25 \%$ de montmorilonita para facilitar a ação de intercalação da argila pela polaridade do anidrido maleico. O masterbatch foi diluído em polipropileno de maneira que as seguintes concentrações de argila fossem obtidas nas amostras de nanocompósitos: $2,5 \%, 5,0 \%, 7,5 \%$ e $10,0 \%$ de argila, identificadas respectivamente, por N2,5, N5, N7,5 e N10.

Além disso, foram processados, sob as mesmas condições, o polipropileno puro e duas formulações contendo polipropileno com $15,0 \%$ e $30,0 \%$ de polipropileno graftizado com anidrido maleico (PP, P15 e P30), respectivamente, concentrações que correspondem às composições contendo $5,0 \%$ e $10,0 \%$ de argila, a fim de verificar a influência da adição de PP-g-AM sobre as propriedades da matriz. As composições das amostras preparadas podem ser visualizadas na Tabela 1.

A análise estrutural foi feita por difração de raios-X com o equipamento Philips, modelo X'Pert, varredura a $2 \theta$ na faixa de $2^{\circ}$ a $10^{\circ}$, com radiação $\mathrm{CuK} \alpha 1,54 \AA$, potência no tubo de

Tabela 1. Composições das amostras preparadas: PP, P15, P30, N2,5, N5, $\mathrm{N} 7,5$ e N10

\begin{tabular}{lccc}
\hline Amostra & $\begin{array}{c}\text { PP } \\
(\boldsymbol{\%})\end{array}$ & $\begin{array}{c}\text { PP-g-AM } \\
(\boldsymbol{\%})\end{array}$ & $\begin{array}{c}\text { Montimorilonita Cloisite 20A } \\
(\boldsymbol{\%})\end{array}$ \\
\hline PP & 100 & - & - \\
P15 & 85 & 15 & - \\
P30 & 70 & 30 & - \\
N2,5 & 90 & 7,5 & 2,5 \\
N5 & 80 & 15 & 5 \\
N7,5 & 70 & 22,5 & 7,5 \\
N10 & 60 & 30 & 10 \\
\hline
\end{tabular}


$40 \mathrm{Kv}$ e corrente de $30 \mathrm{~mA}$. Os ensaios de tração foram realizados de acordo com a norma ASTM D638 ${ }^{[13]} \mathrm{em}$ uma Máquina Universal de Ensaios, marca Emic, Modelo DL2000, com célula de carga de $5000 \mathrm{~N}$ e velocidade da travessa de $50 \mathrm{~mm} / \mathrm{min}$ e os ensaios de impacto foram realizados de acordo com a norma ASTM D256 $6^{[14]}$ método A em uma máquina de Pêndulo de Impacto, marca Ceast, modelo 6545/000, com martelo de Impacto de $2 \mathrm{~J}$.

\section{Resultados e Discussão}

\section{Caracterização estrutural}

A Figura 3 ilustra os resultados obtidos para montmorilonita Cloisite 20A, masterbatch e nanocompósitos. Os picos de difração de interesse na caracterização de nanocompósitos são os que aparecem em regiões entre $2^{\circ}$ a $3^{\circ}$, escala em $2 \theta$. Esses picos são referentes aos espaçamentos basais, ou seja, picos do plano (001) da argila.

Os picos que estão na região de $6^{\circ}$ a $7^{\circ}$ são considerados picos de segunda ordem, conforme relatado na literatura ${ }^{[15]}$. Segundo Santos (1989) ${ }^{[4]}$ em cristalografia de raios-X, não é necessário considerar a reflexão do plano (002) como segunda ordem de (001), uma vez que os planos (002) do retículo são univocamente identificados tal como qualquer outro plano (hkl). Contudo, é comum encontrarem-se referências à reflexão dos planos (002) e (003), como sendo, respectivamente, segunda e terceira ordens do plano (001).

A montmorilonita Cloisite 20A apresentou um pico de difração a $3,74^{\circ}$ que corresponde ao espaçamento basal de $2,36 \mathrm{~nm}$, e um segundo pico a $7,44^{\circ}$ com espaçamento de $1,19 \mathrm{~nm}$. O pico presente a $7,44^{\circ}$ pode ser um pico de segunda ordem da Cloisite 20A. Após o processamento do masterbatch os picos da argila sofreram um deslocamento para $3,50^{\circ} \mathrm{com}$ espaçamento basal de $2,52 \mathrm{~nm}$, e $6,76^{\circ}$, com espaçamento de $1,30 \mathrm{~nm}$, mostrando que ocorreu a intercalação do polipropileno graftizado com anidrido maleico entre as camadas de argila. Para o nanocompósito contendo $2,5 \%$ de argila, foi obtida uma estrutura intercalada, onde os picos de difração passaram para $2,34^{\circ} \mathrm{com}$ espaçamento basal de $3,77 \mathrm{~nm}$, correspondendo a um aumento de $1,41 \mathrm{~nm}$ no espaçamento basal com relação a montmorilonita Cloisite 20A, e o segundo pico a $6,4^{\circ}$, com espaçamento de $1,38 \mathrm{~nm}$. Com o nanocompósito contendo $5,0 \%$ de argila, ocorreu o desaparecimento do primeiro pico de difração, mas o segundo pico foi observado a $6,64^{\circ} \mathrm{com}$ espaçamento de $1,33 \mathrm{~nm}$. Este comportamento sugere que ocorreu esfoliação de uma parte da argila. Lee, Mielewski e Baird (2004 ${ }^{[15]}$ também relatam a existência de picos secundários em seu trabalho. Eles observaram em nanocompósitos de polipropileno e montmorilonita organofílica preparados por extrusão um pico a $3,8^{\circ}$ indicando o espaçamento basal (001) de 23,1 e a ocorrência de um pico secundário a $8,3^{\circ}$, e para nanocompósitos preparados por ultrassonificação à ocorrência de um pico secundário a $5,3^{\circ}$ enquanto o pico característico do espaçamento basal (001) da nanoargila surgiu a $3,7^{\circ}$.

Para o nanocompósito contendo $7,5 \%$ de argila os picos

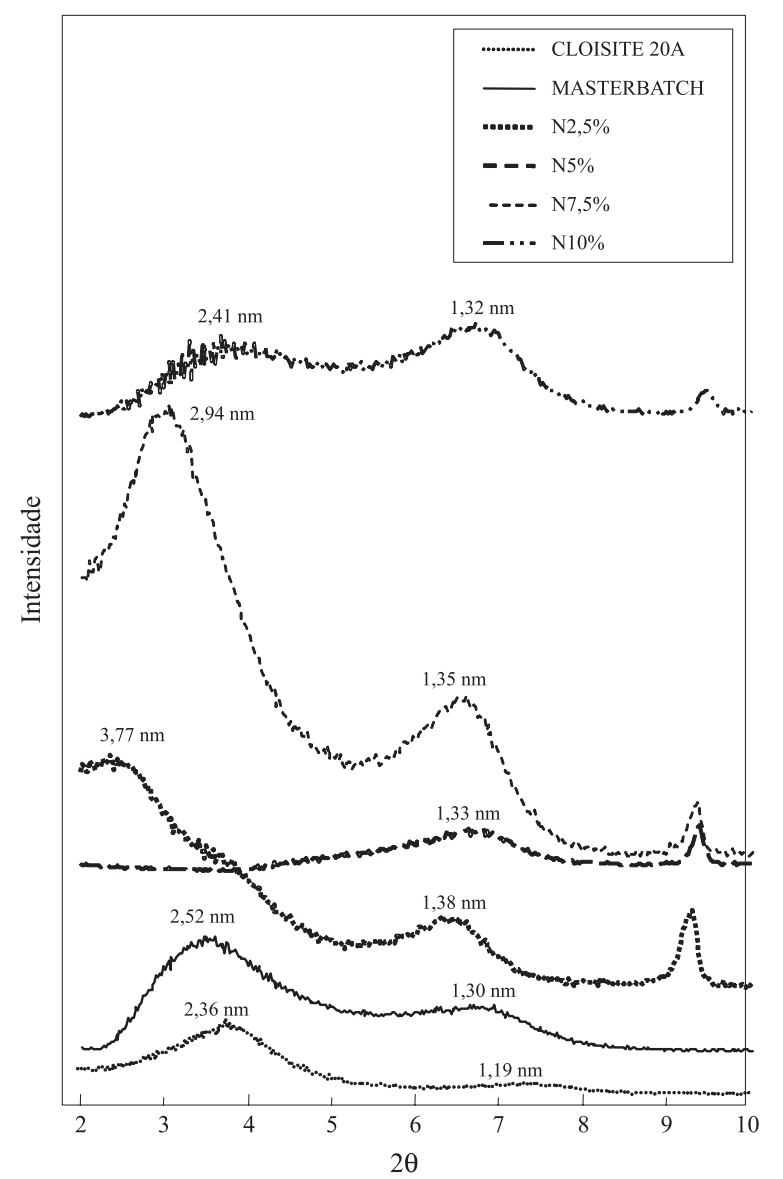

Figura 3. Difratogramas de raios-x da Cloisite 20A, masterbatch, N2,5, N5, N7,5 e N10

foram observados a $3,0^{\circ}$, com espaçamento basal de $2,94 \mathrm{~nm}$ e $6,56^{\circ} \mathrm{com}$ espaçamento de $1,35 \mathrm{~nm}$, enquanto para o nanocompósito contendo $10,0 \%$ de argila esses picos ficaram a $3,66^{\circ}$ com espaçamento basal de $2,41 \mathrm{~nm}$, e $6,70^{\circ} \mathrm{com}$ espaçamento de $1,32 \mathrm{~nm}$. As estruturas obtidas nas composições de $7,5 \%$ e $10,0 \%$ de argila também são estruturas intercaladas, mantendo a estrutura original do masterbatch, porém em N10 o pico sofreu um pequeno deslocamento para $3,66^{\circ}$ enquanto que no masterbatch estava a $3,50^{\circ}$, o que pode ser atribuído à compactação da argila.

Outra hipótese para os picos que se encontram na faixa entre 6 a $7^{\circ}$ é que em uma parte da argila pode ter ocorrido escape do cátion orgânico das camadas de argila durante processamento ou quebra de ligações iônicas entre a superfície dos silicatos da argila e o sal quaternário de amônio devido ao efeito do cisalhamento durante o processamento em extrusora, diminuindo assim espaçamento basal entre as camadas de argila. Esse comportamento foi observado por Lee e Goettler $(2004)^{[16]}$ para um nanocompósito de termoplástico vulcanizado (TVP) com 6\% de montmorilonita Cloisite 30B, porém o pico da argila observado que apresentava $1,8 \mathrm{~nm}$ de espaçamento passou a ter $1,44 \mathrm{~nm}$ no nanocompósito.

\section{Resistência à tração}

Os resultados dos ensaios de tração de PP, P15, P30, N2,5, N5, N7,5 e N10 estão resumidos na Tabela 2. 
Tabela 2. Resultados dos ensaios de tração para PP, P15, P30, N2,5, N5, N7,5 e N10

\begin{tabular}{lccccc}
\hline Amostra & $\begin{array}{c}\text { Módulo Elastico } \\
(\mathbf{M P a})\end{array}$ & $\begin{array}{c}\text { Tensão Máxima } \\
(\mathbf{M P a})\end{array}$ & $\begin{array}{c}\text { Tensão Ruptura } \\
(\mathbf{M P a})\end{array}$ & $\begin{array}{c}\text { Def. Força Máx. } \\
(\%)\end{array}$ & $\begin{array}{c}\text { Def. na Ruptura } \\
(\%)\end{array}$ \\
\hline PP & $335,7 \pm 4,4$ & $34,6 \pm 0,8$ & $17,4 \pm 3,9$ & $23 \pm 0,5$ & $345 \pm 42,9$ \\
P15 & $335,7 \pm 6,1$ & $33,9 \pm 0,4$ & $12,4 \pm 5,8$ & $23 \pm 0,5$ & $712 \pm 35,5$ \\
P30 & $333,1 \pm 6,5$ & $33,0 \pm 0,4$ & $22,4 \pm 3,8$ & $21 \pm 0,6$ & $41 \pm 8,9$ \\
N2,5 & $352,4 \pm 4,2$ & $35,3 \pm 0,2$ & $22,5 \pm 8,0$ & $22 \pm 0,4$ & $38 \pm 4,3$ \\
N5 & $358,7 \pm 3,7$ & $34,4 \pm 0,2$ & $31,1 \pm 0,6$ & $20 \pm 0,3$ & $30 \pm 1,8$ \\
N7,5 & $363,7 \pm 6,7$ & $33,1 \pm 0,3$ & $29,8 \pm 10,4$ & $18 \pm 0,9$ & $18 \pm 1,0$ \\
N10 & $371,4 \pm 8,1$ & $29,9 \pm 0,7$ & $29,8 \pm 0,7$ & $13 \pm 0,7$ & $12 \pm 0,7$ \\
\hline
\end{tabular}

Os valores de módulo de elasticidade encontrados para PP, P15 e P30 foram os mesmos, considerando o erro de medida, o que indica que o PP-g-AM nos teores adicionados não interfere no módulo de elasticidade do PP. Ton-That, Perrin-Sarazin e Cole $(2004)^{[17]}$ também relatam que a presença de polipropileno graftizado com anidrido maleico em matriz de polipropileno não tem efeito significativo sobre as propriedades mecânicas do material. Comparando P15 e amostra N5 ocorreu um aumento de 335,7 MPa para 358,7 MPa, e com P30 e N10 o aumento do módulo de elasticidade foi de 333,1 MPa para 371,4 $\mathrm{MPa}$, valores que representam $7 \%$ e $11 \%$ de aumento no módulo de elasticidade da matriz contendo PP-g-AM com a adição de 5,0 e 10,0\% de argila, respectivamente.

Entre os nanocompósitos foi observado um aumento no módulo de elasticidade em função do aumento da concentração de argila, sendo o menor módulo para N2,5 de 352,4 MPa e o maior para N10 com 371,4 MPa.

Em todas as composições não foram observadas grandes variações na tensão de força máxima, exceto para N10 que teve uma pequena queda para 29,9 MPa comparado a P30 com valor de $33,0 \mathrm{MPa}$, o que pode ser devido à quebra de partículas ou mesmo um empacotamento das camadas de argila, conforme observado nos resultados de difração de raios-x. A tensão de ruptura para PP foi de 17,4 MPa enquanto em P15 foi observada uma ligeira queda para $12,4 \mathrm{MPa}$ e em P30 a tensão aumentou para 22,4 MPa. Comparando P15 e N5 o aumento da tensão de ruptura foi de 12,4 MPa para $31,1 \mathrm{MPa}$ e para P30 e N10 o aumento foi de 22,4 MPa para 29,8 MPa. Considerando o PP puro e as amostras contendo PP e PP-g-AM, P15 e P30, os nanocompósitos apresentaram aumento da tensão de ruptura, sendo que o maior valor obtido foi o de N5 com 31,1 MPa, mas observando os erros experimentais os valores encontrados estão muito próximos.

A deformação na força máxima foi de $23 \%$ em PP e P15 e $21 \%$ para o $\mathrm{P} 30$, o que mostra que o PP-g-AM provoca uma pequena queda da deformação quando adicionado ao PP. Entre P15 e N5 ocorreu uma queda de $23 \%$ para $20 \%$ na deformação, e entre P30 e N10 a queda foi de $21 \%$ para $13 \%$.

A deformação na ruptura do PP foi $345 \%$ e teve um aumento pronunciado para $712 \%$ em P15, porém em P30 essa deformação foi de apenas 41\%. Com relação a P15 e N5 a deformação caiu de $712 \%$ para $30 \%$, e em P30 e N10 a de- formação caiu de $41 \%$ para $12 \%$ com a adição de argila. A queda na deformação na ruptura também foi observada para os nanocompósitos a medida em que a concentração de argila aumentou.

A dispersão das camadas de argila na matriz leva à melhoria no módulo de elasticidade e resistência à tração, o que pode ser atribuído à tenacidade das camadas de silicato que contribuem na formação de fases poliméricas imobilizadas ou parcialmente imobilizadas. Também é possível que a orientação das camadas de argila bem como a orientação molecular contribuam para os efeitos de reforço observados.

\section{Resistência ao impacto}

Os resultados de resistência ao impacto para PP, P15, P30, N2,5, N5, N7,5 e N10 estão na Tabela 3.

A resistência ao impacto ligeiramente melhor com relação a PP puro foi observada para P15, o que indica que a presença de PP-g-AM nesta concentração aumenta a resistência ao impacto, mas para P30 este aumento não é significativo. García-Lopez et al $(2003)^{[18]}$ relataram que a resistência ao impacto é geralmente reduzida com o aumento da concentração de polipropileno graftizado com anidrido maleico. Comparando P15 com N5 é observada uma ligeira queda na resistência ao impacto de $30,7 \mathrm{~J} / \mathrm{m}$ para $28,9 \mathrm{~J} / \mathrm{m}$, enquanto entre P30 e N10 ocorre uma queda mais significativa na resistência ao impacto de $28,1 \mathrm{~J} / \mathrm{m}$ para $19,8 \mathrm{~J} / \mathrm{m}$,

Tabela 3. Resultados de resistência ao impacto para PP, P15, P30, N2,5, $\mathrm{N} 5, \mathrm{~N} 7,5$ e N10

\begin{tabular}{cc}
\hline Amostra & $\begin{array}{c}\text { Resistência ao impacto } \\
(\mathbf{J} / \mathbf{m})\end{array}$ \\
\hline PP & $26,5 \pm 4,3$ \\
P15 & $30,7 \pm 4,8$ \\
P30 & $28,1 \pm 5,5$ \\
N2,5 & $25,2 \pm 4,3$ \\
N5 & $28,9 \pm 5,8$ \\
N7,5 & $23,8 \pm 2,1$ \\
N10 & $19,8 \pm 4,4$ \\
\hline
\end{tabular}


mostrando que a concentração de $10,0 \%$ de argila tornou o material mais frágil.

Entre os nanocompósitos, N5 apresentou resistência ao impacto ligeiramente melhor, o que pode ser atribuído à esfoliação da argila com relação às outras formulações, seguido de N2,5, mas as composições N7,5 e N10 apresentaram queda na resistência ao impacto, devido a maiores concentrações de argila. Considerando os erros experimentais, os valores encontrados estão muito semelhantes, portanto pode ser observado que a formação de nanocompósitos não proporcionou melhorias na propriedade de resistência ao impacto do polipropileno.

Alguns autores relatam que a presença de agente compatibilizante e argila organofílica aumenta a resistência ao impacto do material, possivelmente devido à modificação da morfologia da matriz. Além disso, a argila poderia absorver a energia do impacto e reduzir a propagação de microfissuras ${ }^{[10]}$. Porém isto não é sempre observado. Liu e $\mathrm{Wu}(2001)^{[19]}$ mostraram que resistência ao impacto é praticamente constante em nanocompósitos de polipropileno e montmorilonita modificada com brometo de hexadecil trimetil amônio e co-intercalada com epoxipropil metacrilato em concentrações na faixa de 0 a $7 \%$ de argila.

\section{Conclusões}

A metodologia empregada permitiu obter nanocompósitos de estruturas intercaladas ou parcialmente esfoliadas em função da concentração de argila. O maior espaçamento basal $(3,77 \mathrm{~nm})$, foi observado para a composição contendo $2,5 \%$ de montmorilonita. Com 5,0\% de montmorilonita, o pico associado ao plano (001) da argila desapareceu o que sugere que a argila foi parcialmente esfoliada. As composições contendo $7,5 \%$ e $10,0 \%$ de montmorilonita apresentaram menores espaçamentos basais e isso mostra que aumentando a concentração de argila o processo de intercalação ou esfoliação na matriz polimérica torna-se mais difícil sob as condições de processamento empregadas na preparação dos materiais.

Os valores dos módulos de elasticidade do PP não foram alterados pela adição de PP-g-AM em P15 e P30, mas aumentaram ligeiramente nos nanocompósitos em função do aumento da concentração de montmorilonita, considerandose os erros experimentais. A tensão na força máxima foi praticamente constante em todas as composições, exceto para N10 que teve uma queda no valor, devido ao material ter um comportamento mais frágil (menor deformação de ruptura e menor resistência ao impacto), o que pode ser devido à quebra de partículas ou mesmo empacotamento das camadas de argila, conforme observado nos resultados de difração de raios-x. A tensão de ruptura para os nanocompósitos foi semelhante considerando os erros experimentais, porém aumentou com relação a PP, P15 e P30. O alongamento diminuiu com o aumento da concentração de argila nos nanocompósitos, mostrando que a incorporação de argila em PP tornou o material mais frágil o que pode ser atribuído presença de uma fase parcialmente dispersa e descontinua na estrutura promovendo uma ruptura prematura do corpo-de-prova.

\section{Agradecimentos}

À CAPES e à empresa Cromex SA pelo apoio ao projeto com materiais e infra-estrutura laboratorial para realização dos ensaios.

\section{Referências Bibliográficas}

1. Bourbigot, S.; Devaux, E.; Flambard, X. - Polym. Degrad. and Stab., v. 75, p.397-402 (2002).

2. Alexandre, M.; Dubois, P. - Materials Science and Engineering, v.28, p. 1-63 (2000).

3. Beyer, G. - Plastics Additives \& Compounding, p. 22-28, 2002.

4. Santos, P. S. "Ciência e Tecnologia de Argilas". Ed. Edgard Blucher, São Paulo (1989).

5. Gorassi, G. et al. - Polymer, v. 44, p. 2271-2279 (2003).

6. Zheng, X.; Wilkie, C. A. - Polym. Degrad. and Stab., v. 81, n. 1, p.1-12 (2003).

7. LeBaron, P. C.; Wang, Z.; Pinnavaia, T. J. - Applied Clay Science, v.15, p. 11-29 (1999).

8. Krishnamoorti, R; Yurekli, K. - Current Opinion in Coll. \& Interf. Sci., v. 6, p. 464-470 (2000).

9. Hambir, S.; Bulakh, N.; Jog, J. P. Polym. Eng. and Sci., v.42, n.9, p. 1800-1807 (2002).

10. Yao, H. et al. Polym. Eng. and Sci., v.42, n.9, p. 18081814 (2002).

11. Pantoustier, N. et al. Polym. Eng. and Sci., v.42, n.9, p. 1928-1937 (2002).

12. Stewart, R. Plastics Engineering, p. 22-29 (2004).

13. ASTM D638-99 - "Standard test method for tensile properties of plastics", v. 08.01 (2000).

14. ASTM D256-97 - "Standard test methods for determining the Izod pendulum impact resistance of plastics method A", v.08.01 (2000).

15. Lee, E. C.; Mielewski, D. F.; Baird, R. J. - Polym. Eng. and Sci., v.44, n.9, p. 1773-1782 (2004).

16. Lee, K. Y.; Goetler, L. A. Polym. Eng. and Sci., v. 44, n.6, p.1103-1111, 2004.

17. Ton-That, M.-T.; Perrin-Sarazin, F.; Cole, K. C. - Polym. Eng. and Sci., v. 44, n.7 (2004).

18. García-López, D. et al. - European Polym. Journal, v. 39, p. $945-950$ (2003).

19. Liu, X.; Wu, Q. - Polymer, v. 42, p. 10013-10019 (2001).

Enviado: 07/10/05

Reenviado: 22/02/06

Aprovado: 03/03/06 